\section{Structure du récepteur Smoothened}

\author{
Martial Ruat ${ }^{1}$, Lucile Hoch ${ }^{1}$, Hélène Faure ${ }^{1}$, \\ Didier Rognan²
}

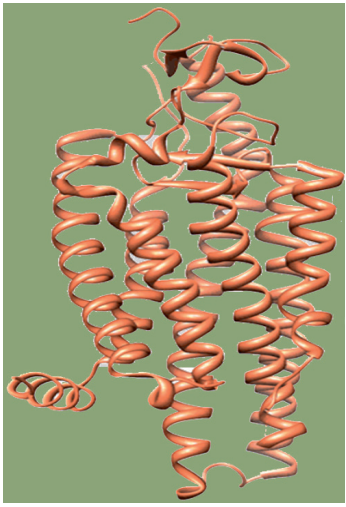

${ }^{1}$ CNRS, Institut de neurobiologie Alfred Fessard, laboratoire de neurobiologie et du développement, UPR 3294, équipe transduction du signal et neuropharmacologie développementale, bâtiment 33 1, avenue de la Terrasse, 91198 Gif-sur-Yvette, France ;

${ }^{2}$ Laboratoire d'innovation thérapeutique, UMR 7200, centre de recherche du médicament MEDALIS, 74 , route du Rhin, 67400 Illkirch, France.

système nerveux central adulte [2]. ruat@inaf.cnrs-gif.fr

Ces observations et d'autres, suggèrent que la voie Hh joue un rôle important dans le maintien des cellules souches cancéreuses [2-5].

Les protéines $\mathrm{Hh}$ interagissent avec le récepteur membranaire Patched (Ptc), levant ainsi l'inhibition que Ptc exerce sur Smo (Figure 1). L'activation de la voie Hh est à l'origine d'une cascade de signalisation intracellulaire complexe qui met en jeu, notamment, les facteurs de transcription de la famille Gli et aboutit à la régulation de l'expression de gènes cibles, tels que Ptc lui-même. Plus récemment, le cil primaire, un organite présent à la surface de la plupart des cellules chez les vertébrés, a également été impliqué dans la transmission du signal Sonic Hedgehog (Shh) in vitro et in vivo [3].

\section{Intérêts thérapeutiques du récepteur Smo dans le traitement de cancers}

Des mutations des gènes $\mathrm{Smo}$ et Ptc sont responsables de tumeurs Un signal Hh aberrant peut induire et maintenir un processus tumoral via plusieurs mécanismes, parmi lesquels des mutations inactivatrices du gène suppresseur de tumeur Ptc ou activatrices de Smo [4-6]. De telles mutations de Smo et de Ptc sont observées dans des tumeurs primitives neuro-ectodermiques du cerveau, notamment des médulloblastomes, et dans des formes sporadiques de carcinome basocellulaire (BCC), une forme de cancer de la peau très fréquente chez l'homme. Des mutations inactivatrices de Ptc sont associées au syndrome de Gorlin, une maladie autosomale dominante caractérisée par des malformations craniofaciales et cérébrales, une incidence élevée de BCC et la présence de médulloblastomes [7]. 


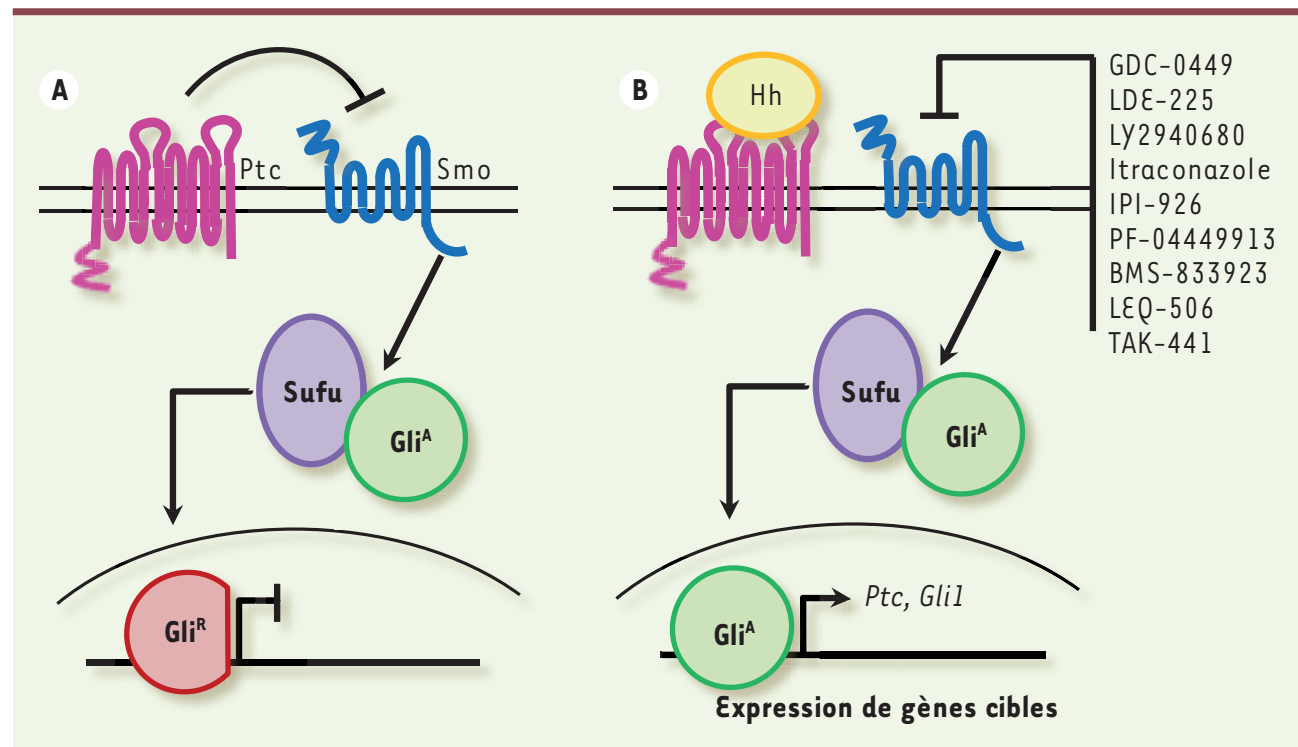

Figure 1. Transduction du signal Hh. A. En l'absence de son ligand, le récepteur Ptc inhibe Smo, un récepteur appartenant à la classe $F$ des RCPG. Le régulateur négatif Sufu (suppressor of fused) interagit avec les formes activatrices des facteurs de transcription Gli $\left(\mathrm{Gli}^{\mathrm{A}}\right)$ associées à la voie Hh et les maintient en dehors du noyau. La forme répressive des facteurs Gli $\left(G l i{ }^{R}\right)$ générée migre dans le noyau et bloque la transcription des gènes cibles. B. En présence de Hh, l'inhibition exercée par Ptc sur Smo est levée. Smo inhibe la formation de Gli ${ }^{R}$ et active Gli ${ }^{A}$ dont la translocation dans le noyau permet d'induire la transcription des gènes cibles de la voie, comme Ptc et Glil. Les antagonistes du récepteur Smoothened testés, ou en cours d'essais cliniques, comme anticancéreux chez l'homme sont indiqués [8]. Ces molécules sont utilisées seules pour le traitement de BCC ou de médulloblastomes associés à des perturbations de la voie Hh, ou en combinaison avec d'autres thérapies pour des tumeurs où la voie Hh est suspectée d'être réactivée.

\section{La réactivation de la voie Hedgehog est associée à des cancers}

La dérégulation de la voie th est observée dans de nombreuses tumeurs, incluant les cancers de la prostate, du pancréas, du poumon, de l'estomac, du sein et les myélomes multiples [4-6]. Elle induit des événements cellulaires incluant une inhibition de l'apoptose et une augmentation de la prolifération via l'expression de cyclines et de kinases. La cellule tumorale est, dans certains cas, à la fois capable de sécréter le ligand Hh et d'y répondre (modèle autocrine). Cependant, le ligand Hh peut aussi agir sur les cellules stromales qui environnent la tumeur et qui expriment le système de réception/transduction du signal, notamment le récepteur Smo (modèle paracrine).

\section{Les antagonistes de Smo en phase clinique pour le traitement} de tumeurs

Smo est considéré comme l'une des principales cibles dont l'inhibition permettrait de bloquer la voie Hh. La preuve de ce concept est apportée par l'existence de plusieurs inhibiteurs de Smo ayant déjà atteint les phases de développement préclinique et clinique (Figures 1 et 2). Certaines de ces molécules ont montré une efficacité dans différents modèles de xénogreffes et ont conduit à la récente mise en place d'essais thérapeutiques de phase I-II dans des tumeurs avancées ou métastatiques $[4,6,8,9]$. Le composé GDC-0449 (vismodegib) de la société Genentech a été le premier antagoniste de Smo introduit récemment sur le marché pour le traitement de formes avancées de BCC. Ce composé a aussi été utilisé par voie orale chez un patient atteint d'une forme métastatique de médulloblastome réfractaire à de multiples thérapies. Malgré une rapide régression de la tumeur et des symptômes associés, le patient a progressivement développé une résistance à la molécule qui s'est révélée être associée à l'apparition d'une mutation ponctuelle transformant le résidu aspartate 473 de Smo en un résidu histidine (D473H). Cette mutation, également identifiée dans un modèle murin de médulloblastomes traité par le GDC-0449, empêche la liaison de la molécule sur Smo, la rendant inactive [10].

Le LDE-225 (société Novartis) est aussi efficace pour le traitement de médulloblastomes murins dépendants de Hh. Cependant, des résistances sont apparues faisant intervenir différents mécanismes, tels qu'une amplification chromosomique d'un gène codant pour l'un des facteurs de transcription associé à la voie Hh (Gli2), des mutations de Smo, ou une régulation positive de la signalisation de la phosphatidylinositol 3-kinase [11]. Le composé Ly2940680 (société Lilly) est un antagoniste puissant, dans différents tests précliniques, de la voie Hh [12]. Il est en développement clinique pour différents cancers où une dérégulation de la voie Hh est suspectée [1]. De nombreux autres composés antagonistes ont été identifiés par des laboratoires pharmaceutiques ou des laboratoires publics en utilisant des techniques de criblage à haut débit [8] ou virtuel basé sur un pharmacophore [13] (Figures 1 et 2). II s'avérait donc particulièrement important d'identifier la structure du récepteur $S m o$ et les sites de reconnaissance des antagonistes. 


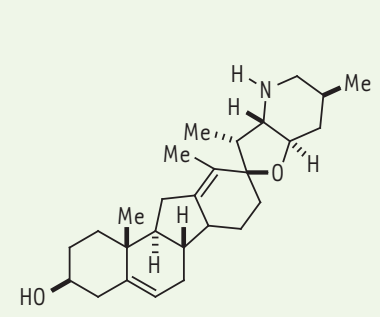

Cyclopamine

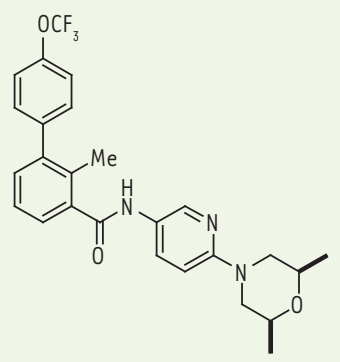

LDE-225

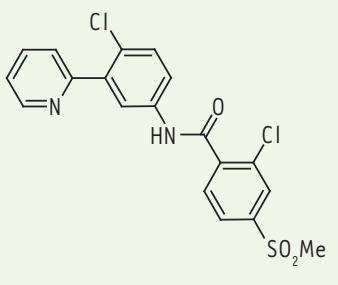

GDC-0449

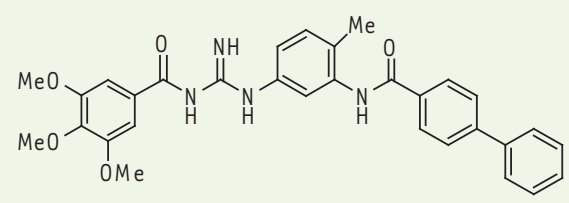

MRT-83

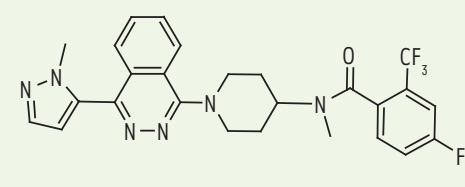

Ly2940680
Figure 2. Structures des antagonistes du récepteur Smoothened. La cyclopamine, un alcaloïde naturel, est le premier antagoniste de Smo à avoir été caractérisé. Les composés LDE-225 (société Novartis), GDC-0449 (Genentech) et Ly2940680 (Lilly) font partie des molécules en essais cliniques (plus de 50 essais ont été programmés) comme agents anticancéreux dans des tumeurs associées à une perturbation de la voie de signalisation Hh. L'acylguanidine MRT-83, issue d'une optimisation de composés identifiés par criblage virtuel d'une banque de molécules [13], est en phase préclinique.

\section{Stratégie utilisée pour purifier le récepteur Smo}

Smo présente des homologies importantes avec les récepteurs Frizzled (FZD) qui transmettent la signalisation des protéines Wnt (winglesstype MMTV integration site), des morphogènes qui jouent un rôle important durant l'embryogenèse. L'homologie faible avec les autres classes de récepteurs couplés aux protéines $G(R C P G)$ a conduit à classifier ces récepteurs dans la classe F des RCPG [14] $(\rightarrow)$ Voir le numéro thématique RCPG, $m / s$ $n^{\circ} 10$, octobre 2012

\section{$(\rightarrow)$.}

Cependant, l'appartenance du récepteur Smo à la famille des RCPG demeurait controversée avant sa cristallisation. Pourtant, ce récepteur peut être phosphorylé par des kinases permettant le recrutement de la $\beta$-arrestine, et peut aussi activer différentes protéines $G[15,16]$.

Ce récepteur est présent en faible abondance dans les tissus, ce qui empêche toute purification en quantité suffisante pour des études de cristallisation. Afin de le cristalliser, les chercheurs ont tout d'abord surexprimé cette protéine dans un système hétérologue. En effet, la production d'un récepteur membranaire fonctionnel s'avère une étape essentielle, et l'expression dans des bactéries ou encore dans des levures présente plusieurs avantages [17]. Des premières études d'expression de Smo, mais aussi de Ptc, dans la souche Saccharomyces cerevisiae de levure avaient permis de démontrer que ces protéines pouvaient être produites en quantité importante en gardant leur caractère fonctionnel [18]. Cependant, l'expression dans des cellules d'insecte de RCPG, tels que les récepteurs $\beta_{1}$ - et $\beta_{2}$-adrénergiques, et les récepteurs de l'adénosine $\left(A_{2 A}\right)$, des chimiokines (CXCR4), de la dopamine $\left(\mathrm{D}_{3}\right)$ ou de la sérotonine $\left(5-\mathrm{HT}_{1 \mathrm{~b}}, 5-\mathrm{HT}_{2 \mathrm{~b}}\right)$ s'est révélée particulièrement avantageuse, conduisant à leur purification et leur cristallisation [17, 19-21].

Afin d'assurer une expression élevée et d'augmenter les chances de cristallisation de Smo, les parties amino- et carboxy-terminales du récepteur ont été modifiées. En effet, la partie aminoterminale extracellulaire $(\varepsilon C D)$ contient un domaine riche en cystéine (CRD) connecté au premier domaine transmembranaire (TMl) par une quarantaine d'acides aminés dont trois cystéines (C193, C213 et C217). Ces cystéines sont strictement conservées dans la séquence des récepteurs FZD, ce qui suggère qu'elles joueraient un rôle particulier dans le maintien de l'intégrité structurale des boucles extracellulaires $(\varepsilon C L)$ du récepteur. Cette quarantaine d'acides aminés a ainsi été préservée et le CRD remplacé par une protéine de fusion thermostable dérivée de l'apocytochrome et déjà utilisée pour la cristallisation de RCPG [1]. La partie carboxyterminale intracellulaire longue de plus de 200 acides aminés, proposée comme jouant un rôle de rhéostat lors de l'activation du récepteur en étant phosphorylée par différentes kinases [22], a été tronquée afin de faciliter la cristallisation du récepteur. Cette forme tronquée du récepteur Smo exprimée dans les cellules d'insectes sf9, se lie à la cyclopamine avec une affinité comparable à celle du récepteur Smo sauvage exprimée dans des cellules eucaryotes HEK293T. La cyclopamine, un alcaloïde qui peut être extrait d'une plante appelée vératre blanc, a été le premier antagoniste du récepteur Smo à avoir été identifié (Figure 2). Sous sa forme tritiée, c'est un ligand radioactif qui permet de marquer le récepteur Smo. II peut être ainsi utilisé dans des expériences de compétition pour démontrer qu'un autre antagoniste de Smo, le composé Ly2940680, conserve les mêmes propriétés de liaison vis-à-vis du récepteur muté et du récepteur sauvage. Le récepteur Smo tronqué a ainsi été purifié en présence de Ly2940680 afin 


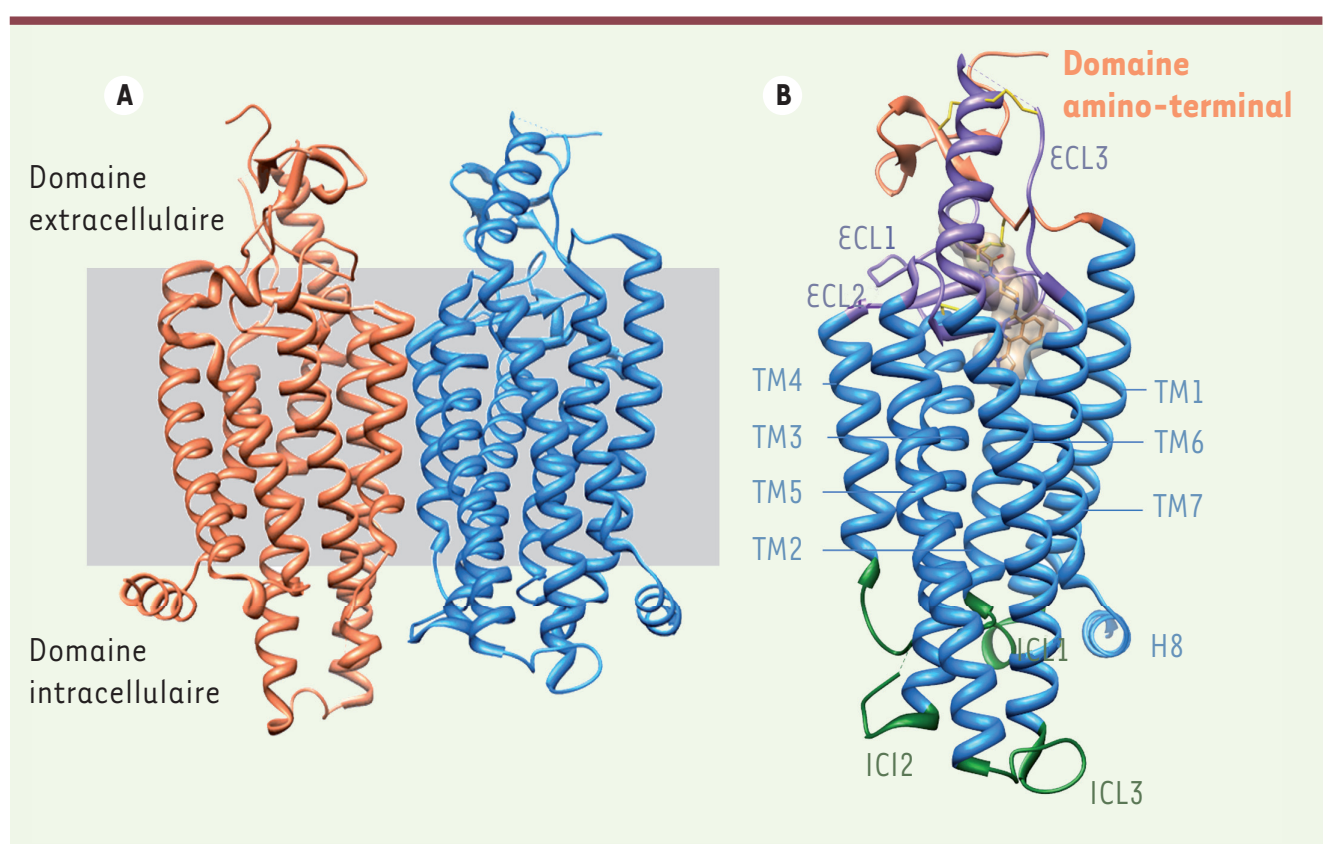

Figure 3. Structure cristallographique du récepteur Smoothened. A. Structure du dimère cristallographique (monomère $A$ en bleu, monomère $B$ en orange). Les contours d'une membrane cellulaire sont représentés en gris. $\boldsymbol{B}$. Structure du monomère: Smoothened présente une structure prototypique d'un RCPG à sept hélices transmembranaires (TMI-7, bleu), un domaine aminoterminal extramembranaire (orange), trois boucles extracellulaires ( $\varepsilon \mathrm{CL} 1-3$, violet), trois boucles intracellulaires (ICLI-3, vert), et une huitième hélice (cyan) carboxy-terminale. Pour des raisons d'ingénierie moléculaire, la partie amino-terminale a été partiellement tronquée afin de favoriser la cristallisation de la protéine dans un environnement lipidique. L'anticancéreux Ly2940680 est représenté au niveau de sa poche de liaison (surface jaune transparente) localisée au niveau de la face externe des 7TM. Smoothened montre néanmoins une très grande particularité dans la structure 3D des segments extracellulaires, notamment un réseau de quatre ponts disulfures (bâtons jaunes) contribuant à rigidifier la structure du récepteur et à tapisser le haut du site de reconnaissance des antagonistes.

d'augmenter la stabilité du complexe, ce qui a permis de le purifier et de déterminer sa structure tridimensionnelle par diffraction aux rayons $X$. La structure de Smo couplé à un agent antitumoral a ainsi pu être résolue avec une résolution de $2,5 \AA$.

\section{Le récepteur Smo, un récepteur à sept domaines transmembranaires}

L'organisation structurale de Smo correspond à celle d'un RCPG avec la présence de 7TM connectés par trois $\varepsilon C L(\varepsilon C L l-3)$. La partie amino-terminale connectant l' $E C D$ avec les $7 T M$, ainsi que les $\varepsilon C L 1-3$, forment un arrangement complexe stabilisé par quatre ponts disulfures (Figure 3 ). II existe ainsi un pont entre les cystéines $\mathrm{C} 193$ et C213 présentes dans la partie amino-terminale ; un deuxième entre les cystéines C217 et C295, entre ce même domaine et l' $\varepsilon C L 1$; un troisième reliant les cystéines C314 et C390 localisées au niveau de l'ECL1 et l'દCL2, respectivement; et, enfin, un quatrième entre les cystéines C490 et C507 situées dans l'ECL3. L'antagoniste Ly2940680 se lie dans une poche localisée dans la partie extracellulaire délimitée par les 7TM et les 3 عCL.

\section{Le récepteur Smo forme un dimère}

Le récepteur cristallise sous forme d'un dimère asymétrique, bien que la différence entre les deux protomères soit faible, avec des interfaces mettant en jeu les hélices 4 et 5 , ce qui a été aussi observé pour le récepteur CXCR4. De nombreux RCPG s'associent pour former des dimères, voire des oligomères. Ces études, très souvent réalisées dans des cellules eucaryotes surexprimant ces récepteurs, sont particulièrement nombreuses et ont permis de mieux appréhender le rôle de chaque protomère lors de l'activation ou de l'inactivation de ces récepteurs, comme dans le cas des récepteurs de la classe C des RCPG [23, 24]. Les données concernant l'existence et le rôle fonctionnel de ces dimères sont cependant relativement peu nombreuses. De telles études ont pourtant été rapportées pour le récepteur Smo de drosophile génétiquement modifié, puis exprimé dans l'aile. Des expériences de transfert d'énergie par résonance (FRET, fluorescence resonance energy transfer) ont permis de mettre en évidence la proximité spatiale de deux récepteurs Smo. La protéine Smo de vertébrés forme aussi des dimères en culture cellulaire. Il conviendra de déterminer si la formation de tels dimères, voire d'oligomères qui ont aussi été décrits [25, 26], participe aux phénomènes de coopérativité observés dans des expériences pharmacologiques $[5,6,8,9]$.

\section{Comparaison des 7TM de Smo à ceux de la classe A des RCPG}

Le récepteur Smo, tout comme les récepteurs FZD, présente une homologie de séquence assez faible avec les 
récepteurs $R C P G$ de la classe $A$ : il ne possède pas, par exemple, de motif $D[\varepsilon] R y$ rencontré dans I'hélice III de ces derniers. Cependant, la comparaison de la structure cristallographique de Smo avec celle des récepteurs de la classe $A$, précédemment déterminée, fait apparaître une conservation spatiale relativement élevée, comme par exemple I'orientation de I'hélice VIII parallèle à la surface intracellulaire de la membrane plasmique. II existe pourtant des différences notables qui concernent notamment les résidus proline impliqués dans l'activation des récepteurs de la classe $A$ et localisés dans les hélices V, VI et VII $\left(P^{5.50}, P^{6.50}\right.$ et $\left.P^{7.50}\right)$. L'absence de ces résidus à ces positions dans le récepteur Smo suggère que le mouvement de ces hélices, lors de l'activation, est le résultat de contraintes mettant en jeu d'autres acides aminés qui pourraient être des résidus glycine présents dans ces hélices.

\section{La poche de liaison des antagonistes du récepteur Smo}

L'organisation structurale des sites de liaison du Ly2940680 avec le récepteur Smo est indiquée dans la Figure 4. La poche de liaison de l'antagoniste localisée sur la face externe des 7TM est étendue mais étroite, et entre en contact avec la partie amino-terminale du récepteur, ainsi qu'avec l'ECL2 et l'£CL3. Une ouverture serait ainsi délimitée permettant l'accessibilité de l'antagoniste à la cavité située au niveau des 7TM. Un résidu arginine R4005.39, présent sur la face externe de I'hélice 5 , formerait des liaisons hydrogènes avec le noyau phthalazine du Ly2940680. II est à noter que plusieurs molécules d'eau sont présentes dans la poche de liaison, mais ne semblent pas être en contact direct avec le Ly2940680. Elles pourraient, cependant, participer à la conformation globale et à la dynamique de la poche via des liaisons hydrogènes avec différents résidus la formant, comme par exemple le résidu $\mathrm{D} 473^{6.55}$. C'est la mutation de ce résidu en histidine qui conduit à des mécanismes de résistance chez l'homme traité par le GDC-0449 [10], suggérant un rôle important de ces liaisons hydrogènes pour la fixation de certains antagonistes.

\section{Smo présente des domaines extracellulaires atypiques}

Les boucles $\varepsilon \mathrm{CLl}-3$ de Smo sont particulièrement longues comparées à celles des RCPG de la classe A déjà cristallisés. Avec le domaine connectant I' $€ C D$ aux 7TM, elles forment un chapeau recouvrant le site de liaison de l'antagoniste. L'ECLl est partagée en deux segments distincts délimités par un pont disulfure, alors que l'ECL2 est positionnée à l'intérieur de la cavité formée par les 7TM et interagit directement avec l'antagoniste, participant très largement à la poche de liaison. $\varepsilon n$ accord avec cette hypothèse, des résistances liées à des mutations dans l'ECL2 du récepteur Smo de rongeur ont été observées après des traitements par un inhibiteur de Smo, le composé NVP-LDE225 [27]. Cela suggère que cet antagoniste partage aussi, en partie, la poche de liaison du LY2940680. L'ECL3, la plus longue des trois boucles, forme une protrusion à partir des 7TM dans l'espace extracellulaire. L'hélice du TM6 semble ainsi se prolonger, alors qu'un pont disulfure pourrait participer à la rigidité de la structure. L'ECL3 interagit aussi directement avec plusieurs résidus de l'ECD. L'intégrité de la structure formée

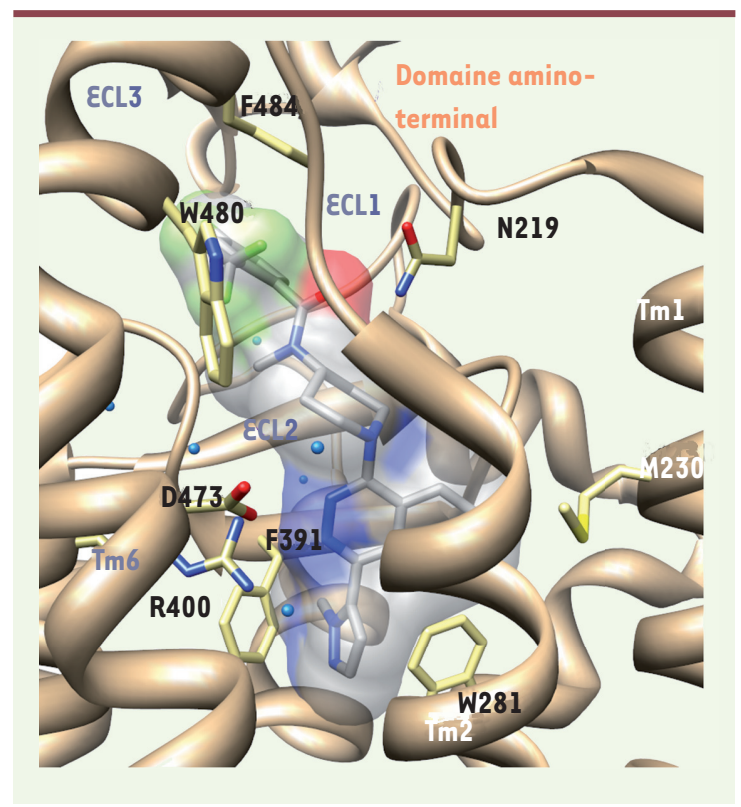

Figure 4. Poche de liaison de l'antagoniste Ly2940680 dans la structure inactivée du récepteur Smoothened (rubans bruns). L'antagoniste est représenté en modèle moléculaire à bâtons (carbone : blanc; azote : bleu; oxygène : rouge ; fluor : vert) dans sa surface moléculaire translucide (même code couleur). Les chaînes latérales des principaux acides aminés de Smoothened au contact de l'antagoniste sont également représentées en modèle à bâtons (même code couleur sauf pour les atomes de carbones en jaune clair). Les domaines transmembranaires (TM) et boucles extracellulaires ( $E C L$ ) formant le site de liaison sont indiqués en violet. Certaines molécules d'eau probablement fixes (balles cyan) participent à la définition de la poche de liaison.

par les $\varepsilon C L 1-3$ est vraisemblablement essentielle pour maintenir Smo dans une conformation inactive.

\section{Conclusion et perspectives}

Tous les antagonistes de Smo se fixent-ils à l'intérieur de la poche de liaison décrite ci-dessus? Le composé Ly2940680 inhibe la liaison de la cylopamine tritiée, suggérant que ces deux composés partagent le même site [1]. Cependant, des données pharmacologiques récentes indiquent que Smo présente plusieurs sites de liaison à de petites molécules. L'impossibilité de démontrer une interaction directe de certaines molécules avec ce récepteur n'exclut pas qu'elles interagissent avec lui $[8,9]$. C'est probablement le cas pour le composé antifongique, itraconazole, proposé comme un antagoniste de Smo [28] et qui a été évalué en clinique pour le traitement de tumeurs dépendantes de Hh [9]. Récemment, le composé GSA-10, appartenant à une nouvelle classe 
d'agonistes de Smo découverte par criblage virtuel, a permis de mettre en évidence de nouveaux états conformationnels de Smo plus ou moins sensibles aux antagonistes connus de Smo [29]. Plusieurs composés de référence, tels que le GDC-0449 et la cyclopamine, se sont révélés des antagonistes peu puissants vis-à-vis de la réponse induite par le GSA10 dans des tests de différenciation cellulaire. D'autres composés, tels que le MRT-83 ou le LDE-225, sont à l'inverse de puissants inhibiteurs. Ces travaux et d'autres $[8,9,30]$ suggèrent qu'il existe vraisemblablement une grande variabilité conformationnelle du récepteur Smo.

Les récents travaux de cristallisation de cette protéine [1] ont donc d'importantes implications pour le développement de nouveaux agents anticancéreux, mais aussi pour d'autres pathologies dans lesquelles l'inhibition de Smo a été proposée comme présentant un intérêt thérapeutique [31, 32]. L'identification des sites de liaison de ces molécules sur le récepteur Smo devrait aussi permettre de mieux comprendre les mécanismes qui gouvernent la régulation de cette voie de signalisation par des molécules endogènes, comme par exemple des dérivés d'oxystérol récemment proposés comme modulateurs allostériques de Smo [33]. $\diamond$

\section{SUMMARY}

Structure of the Smoothened receptor

The Smoothened (Smo) receptor is a major component involved in signal transduction of the Hedgehog ( $\mathrm{Hh}$ ) morphogens both during embryogenesis and in the adult. Smo antagonists represent a promising alternative for the treatment of cancers linked to abnormal Hh signalling. The crystal structure of the human Smo receptor bound to an antitumour agent demonstrates that this receptor belongs to the superfamily of $\mathrm{G}$-protein coupled receptors. The antagonist binds to a pocket localized at the extracellular side formed by the seven transmembrane domains and the complex arrangement of the unusually long extracellular loops. The structure of the Smo receptor will promote the development of small molecules interacting with a key therapeutic target with interests in regenerative medicine and cancer. $\diamond$

\section{LIENS D'INTÉRÊT}

Les auteurs déclarent n'avoir aucun lien d'intérêt concernant les données publiées dans cet article.

\section{RÉFÉRENCES}

1. Wang $\mathrm{C}$, Wu H, Katritch V, et al. Structure of the human smoothened receptor bound to an antitumour agent. Nature $2013 ; 497: 338-43$.

2. Ruat $M$, Angot $\varepsilon$, Traiffort $\varepsilon$. Sonic Hedgehog, un morphogène en quête de fonction dans le cerveau adulte. Med Sci (Paris) $2011 ; 27: 979-85$.

3. Ruat M, Roudaut H, Ferent J, Traiffort $\varepsilon$. Hedgehog trafficking, cilia and brain functions. Differentiation $2012 ; 83$ : S97-104.

4. Scales SJ, de Sauvage FJ. Mechanisms of Hedgehog pathway activation in cancer and implications for therapy. Trends Pharmacol Sci $2009 ; 30: 303-12$.

5. Ng JM, Curran T. The Hedgehog's tale: developing strategies for targeting cancer. Nat Rev Cancer $2011 ; 11: 493-501$.

6. Lin TL, Matsui W. Hedgehog pathway as a drug target: Smoothened inhibitors in development. Onco Targets Ther $2012 ; 5: 47-58$.

7. Bourdeaut F, Pouponnot C, Ayrault 0 . Les médulloblastomes et leurs cellules d'origine. Med Sci (Paris) $2012 ; 28: 805-9$.

8. Queiroz KC, Spek CA, Peppelenbosch MP. Targeting Hedgehog signaling and understanding refractory response to treatment with Hedgehog pathway inhibitors. Drug Resist Updat 2012 ; 15 : 211-22.
9. Hadden MK. Hedgehog pathway inhibitors: a patent review (2009-present) Expert Opin Ther Pat $2013 ; 23: 345-61$.

10. Yauch RL, Dijkgraaf GJ, Alicke B, et al. Smoothened mutation confers resistance to a Hedgehog pathway inhibitor in medulloblastoma. Science $2009 ; 326: 572-4$.

11. Buonamici S, Williams J, Morrissey M, et al. Interfering with resistance to smoothened antagonists by inhibition of the PI3K pathway in medulloblastoma. Sci Transl Med $2010 ; 2: 51-70$.

12. Bender $M$, Hipsking $P$, Capen $A$, et al. Identification and characterization of a novel smoothened antagonist for the treatment of cancer with deregulated hedgehog signaling Cancer Res $2011 ; 71: 2819$.

13. Roudaut $H$, Traiffort $\varepsilon$, Gorojankina T, et al. Identification and mechanism of action of the acylguanidine MRT-83, a novel potent Smoothened antagonist. Mol Pharmacol $2011 ; 79$ : 453-60.

14. Schulte $G$. International union of basic and clinical pharmacology. LXXX. The class Frizzled receptors. Pharmacol Rev $2010 ; 62: 632-67$.

15. Riobo NA, Lu K, Ai X, et al. Phosphoinositide 3-kinase and Akt are essential for Sonic Hedgehog signaling. Proc Natl Acad Sci USA 2006 ; 103 : 4505-10.

16. Masdeu C, Faure H, Coulombe J, et al. Identification and characterization of Hedgehog modulator properties after functional coupling of Smoothened to G15. Biochem Biophys Res Commun 2006 ; 349 : 471-9.

17. Banères JL, Mouillac $B$. Manipulation des récepteurs couplés aux protéines $\mathrm{G}$ : expression, purification et stabilisation in vitro. Med Sci (Paris) 2012 ; $28: 837-44$.

18. Nehme $R$, Joubert 0 , Bidet $M$, et al. Stability study of the human $G$-protein coupled receptor, Smoothened. Biochim Biophys Acta $2010 ; 1798: 1100-10$.

19. Wang $C$, Jiang $Y, M a J$, et al. Structural basis for molecular recognition at serotonin receptors. Science $2013 ; 340: 610-4$.

20. Wacker D, Wang C, Katritch V, et al. Structural features for functional selectivity at serotonin receptors. Science $2013 ; 340: 615-9$.

21. Granier S. Structure des récepteurs mu et delta des opiacés. Med Sci (Paris) $2012 ; 28: 870-5$.

22. Chen $Y$, Jiang J. Decoding the phosphorylation code in Hedgehog signal transduction. Cell Res $2013 ; 23: 186-200$.

23. Ruat $M$, Petrel C. Comment moduler l'activité du récepteur du calcium extracellulaire? Med Sci (Paris) $2004 ; 20: 980-5$.

24. Kniazeff J, Pin JP. Des dimères et des oligomères de récepteurs couplés aux protéines $G$, oui mais pourquoi ? Le récepteur $G_{A B A}$ sous interrogatoire. Med Sci (Paris) $2012 ; 28: 858-63$.

25. Zhao $Y$, Tong $C$, Jiang J. Hedgehog regulates smoothened activity by inducing a conformational switch. Nature $2007 ; 450: 252-8$.

26. Shi D, Lv X, Zhang Z, et al. Smoothened oligomerization/higher order clustering in lipid rafts is essential for high hedgehog activity transduction. J Biol Chem 2013 ; 288 : 12605-14.

27. Dijkgraaf GJ, Alicke B, Weinmann L, et al. Small molecule inhibition of GDC0449 refractory smoothened mutants and downstream mechanisms of drug resistance. Cancer Res $2011 ; 71: 435-44$.

28. Kim J, Tang Jy, Gong R, et al. Itraconazole, a commonly used antifungal that inhibits Hedgehog pathway activity and cancer growth. Cancer Cell 2010 ; 17 : 388-99.

29. Gorojankina T, Hoch L, Faure H, et al. Discovery, molecular and pharmacological characterization of GSA-10, a novel small-molecule positive modulator of Smoothened. Mol Pharmacol $2013 ; 83$ : 1020-9.

30. Teperino R, Amann S, Bayer M, et al. Hedgehog partial agonism drives Warburg-like metabolism in muscle and brown fat. Cell $2012 ; 151$ : 414-26.

31. Horn A, Palumbo K, Cordazzo C, et al. Hedgehog signaling controls fibroblast activation and tissue fibrosis in systemic sclerosis. Arthritis Rheum 2012 ; $64: 2724-33$.

32. Lin AC, Seeto BL, Bartoszko JM, et al. Modulating hedgehog signaling can attenuate the severity of osteoarthritis. Nat Med 2009 ; 15 : 1421-5.

33. Nachtergaele S, Mydock LK, Krishnan K, et al. Oxysterols are allosteric activators of the oncoprotein Smoothened. Nat Chem Biol 2012 ; 8 : 211-20.
TIRÉS À PART

M. Ruat 